\title{
Le corps en vue - trois images du corps chez Michel Houellebecq
}

\author{
Mads Anders Baggesgaard
}

\author{
Université d'Aarhus, Danemark
}

\begin{abstract}
On a souvent taxé de pornographiques les descriptions que Michel Houellebecq fait du corps. Un examen plus attentif nous montre toutefois que Houellebecq ne cherche pas à stimuler sexuellement le lecteur, mais emploie plutôt l'image pornographique dans la perspective d'explorer les possibles et les limites de la description corporelle. A partir du court récit Lanzarote, cet article identifie trois types de descriptions du corps dans l'écriture de Houellebecq, tout d'abord le tableau pornographique, puis la description sensuelle et enfin la description dépersonnalisée d'un futur post-humain dans La Possibilité d'une île, pour montrer que la manière dont Houellebecq traite la problématique s'inscrit dans la tradition réaliste française tout en s'en détachant.
\end{abstract}

\section{Pornographie et visualité}

On a souvent accusé Michel Houellebecq de misogynie et d'objectivation du corps féminin. Ses romans ont sans doute un caractère pornographique : il est rare qu'un chapitre s'écoule sans référence aux organes génitaux. On peut ainsi affirmer sans trop de difficulté que ses descriptions explicites participent à l'apparente sexualisation de l'art et de la sphère publique. Néanmoins, les accusations d'objectivation ne semblent pas toujours fondées. Les descriptions pornographiques de Houellebecq ne sont jamais pure pornographie ; elles sont accumulation consciente d'images et de clichées pornographiques pour permettre l'examen des conditions de la sexualité humaine dans la société d'aujourd'hui. En fait, l'emploi de clichés pornographiques s'inscrit dans une longue tradition littéraire dont le but principal est la description du corps féminin. Cette tradition remonte au réalisme français du XIX ${ }^{\mathrm{e}}$ siècle. L'emploi du tableau pornographique de Houellebecq attire l'attention sur la relation entre vision, sexualité et corps; une relation qu'examine Houellebecq dans l'ensemble de son œuvre dans une tentative de créer une nouvelle image du corps sexuel humain. 
Peut-être l'emploi du tableau pornographique est-il le plus évident dans le récit Lanzarote, qui raconte le séjour estival d'un jeune homme sur cette île. Les vacances du personnage principal culminent lorsque, sur une plage déserte, il prend part à la réalisation d'un fantasme pornographique classique avec deux lesbiennes larges d'esprit. La description de leurs ébats, qui s'étend sur plusieurs pages, est de toute évidence inspirée de l'imagerie pornographique, comme dans ce dernier passage :

Elle dirigea ma queue vers la poitrine de Barbara et recommença à branler par petits coups très vifs, ses doigts en anneau à la racine du gland. Barbara me regarda et sourit; au moment où elle pressa ses mains sur le côté de ses seins pour accentuer leur rondeur, $\mathrm{j}$ 'éjaculai violemment sur sa poitrine. J'étais dans une espèce de transe, je voyais trouble, c'est comme un brouillard que je vis Pam étaler le sperme sur les seins de sa compagne. Je me rallongeai sur le sable, épuisé ; je voyais de plus en plus trouble. Pam commença à lécher le sperme sur les seins de Barbara. Ce geste était infiniment touchant; $j$ 'en eus les larmes aux yeux ${ }^{1}$.

La description correspond aux caractéristiques que l'historien de l'art danois, Rune Gade, propose dans son ouvrage, Staser, sur l'imagerie pornographique. Staser est une étude très poussée de l'histoire de l'image pornographique, des tableaux érotiques du XIX siècle aux récits hard-core du $\mathrm{XX}^{\mathrm{e}}$. Gade considère la pornographie comme une mise en scène complexe de regards. Le regard de la femme est essentiel parce qu'il reflète le regard contemplatif auquel elle s'expose : "Elle "expose" son savoir par l'anticipation du regard de l'observateur : si quelque chose est "dévoilé", c'est le regard désirant de l'observateur, qui est ici défié par la connaissance (phallique) de la femme ${ }^{2} »$. Le regard de la femme révèle qu'elle se sait observée, et, ainsi, que son désir est comparable au désir de l'observateur masculin.

Cette description de l'imagerie pornographique est en accord avec le passage extrait de Lanzarote cité plus haut. Il ne reste rien à l'imagination, tout est visible, jusqu'au money-shot final, quand l'homme éjacule non pas dans la femme, mais sur la femme, exposant le liquide séminal au regard de l'observateur. La vue joue ici un rôle

\footnotetext{
${ }^{1}$ Michel Houellebecq, Lanzarote, Paris, Flammarion, 2000, p. 58.

${ }^{2}$ Rune Gade, Staser - Teorier om det fotografiske billedes ontologiske status \& Det pornografiske tableau, Århus, Passepartout's Særskriftserie, 1997, p. 192. (Ma traduction.)
} 
déterminant, qui est souligné par les jeux de regards entre les amants. Les femmes regardent le personnage principal : Barbara me regarda et sourit, mais s'exposent tout d'abord à son regard, qui est clair jusqu'au moment de l'éjaculation où il devient trouble au point que le narrateur ne peut plus prendre plaisir au spectacle. Les deux femmes s'exposent, montrent leur désir et affirment de cette manière le désir du personnage principal, désir confirmé par l'éjaculation :

L'éjaculation exprime le point où le regard de l'homme trouve le repos en ce qu'il est définitivement cédé à la femme. Le récit y prend fin car le regard est épuisé, laissé sur la femme, qui à partir de ce moment le détient, c'est-à-dire que le désir de la femme est aussi satisfait par la spermatisation. $^{3}$

$\mathrm{Au}$ moment de l'éjaculation, le regard masculin est cédé à la femme, ce qui est souligné par l'ingestion orale de la semence, et comme cela le regard masculin s'épuise. Ainsi, le regard du personnage principal se trouble, et il retombe passif dans le sable; le regard brouillé par des larmes de bonheur.

Cette scène réalise tous les fantasmes sexuels qu'entretenait le personnage principal avant son départ. Les jeux de regards intensifient le désir qui culmine dans la satisfaction absolue. Mais cet état extatique ne dure pas. En effet, les ébats amoureux se déroulent en l'absence d'un quatrième membre du groupe, Rudi, qui ne désire pas prendre part. On en découvre la raison à la fin du récit, dans une lettre que $\mathrm{Ru}-$ di laisse derrière lui au moment de son départ pour adhérer au culte azraëlien. La réticence de Rudi n'est pas due à des scrupules moraux, mais est la conséquence du fait que sa femme l'a quittée pour devenir musulmane à la suite d'une sursaturation de leur vie sexuelle très active. Autrefois ils fréquentaient souvent des boîtes échangistes, mais la nécessité croissante de stimulation visuelle finit par les amener à n'être plus que de simples spectateurs :

Nous en sommes venus à des situations humiliantes où nous nous contentions d'assister en spectateurs passifs aux exhibitions de monstres sexuels parfaits dont nous ne pouvions plus faire partie, vu notre âge. ${ }^{4}$

\footnotetext{
${ }^{3}$ Ibid., p. 219.

${ }^{4}$ Michel Houellebecq, Lanzarote, op. cit., p. 69.
} 
Cette description introduit un trait décisif de la sexualité telle qu'elle est décrite chez Michel Houellebecq: l'expérience qu'une sexualité basée sur la visualité implique la suppression de soi ${ }^{5}$. Pour avoir la certitude du désir de l'autre on exige des manifestations de plus en plus marquées, un processus qui mène la sexualité au-delà des limites du corps. Et la conscience seule de cette relation conduit à un relâchement de la tension sexuelle entre le personnage principal et ses amies lesbiennes autant que plusieurs des personnages de Houellebecq se trouvent castrés à la suite d'affaires sexuelles qui ont mal tourné. Par l'emploi du tableau pornographique, Houellebecq avance une critique de la visualité et met en évidence le processus par lequel une sexualité basée sur la visualité appelle nécessairement à une explicitation toujours plus grande; ou, comme le dit Linda Williams dans son étude sur la pornographie Hard Core : "If sex is the problem, then in hard core more, better or different sex is the solution ${ }^{6} »$. Cependant, comme l'étude de photographies médicales des parties génitales humaines le prouve, la sexualité absolument explicite n'est pas une alternative, et la sexualité visuelle mène donc invariablement à la rupture.

La critique de Houellebecq peut être vue comme le résultat de l'adaptation d'un problème fondamental de l'histoire littéraire : celui de la description du corps humain. Houellebecq appartient en cela à la tradition réaliste française. L'écrivain américain Peter Brooks décrit ainsi dans Body Work l'obsession des réalistes français pour la description du corps féminin nu. De Balzac, à Zola, en passant par Flaubert, le corps féminin est objet de désir, un fétiche qui, parce qu'indescriptible, devient le point fixe de la narration, comme on le voit, par exemple, dans Madame Bovary et Nana. Chez Flaubert, des fragments épars se substituent à la description du corps, allusions métonymiques suggérant la nudité, on mentionnera à ce titre la fameuse " main nue ${ }^{7}$ ", qui apparaît de la fenêtre de la voiture dans laquelle

\footnotetext{
${ }^{5}$ Le terme visualité sert comme traduction du terme anglais visuality utilisé primairement par 1'historien de 1'art Martin Jay dans Downcast Eyes: The Denigration of Vision in Twentieth-Century French Thought, (1993) Berkeley/Los Angeles/London : University of California Press. Visualité décrit ici la sorte particulière de connaissance fondée sur la vue et le regard qui a prédominé dans les sociétés occidentales depuis le siècle des lumières.

${ }^{6}$ Linda Williams, Hard Core: Power, Pleasure, and the "Frenzy of the Visible", Berkeley/Los Angeles, University of California Press, 1989, p. 228.

${ }^{7}$ Gustave Flaubert, Madame Bovary, Paris, Gallimard, 2001, p. 328.
} 
Emma trompe son mari avec Léon. Même chez Zola, qui est beaucoup moins pudique, la nudité de Nana est voilée : « un duvet de rousse faisait de son corps un velours ${ }^{8} »$. Cependant, cette discrétion n'est pas l'effet de pudeur, mais, comme l'indique Brooks, une nécessité, pour que le désir qui pousse la narration se soutienne :

Zola's narratives of unveiling the female body sooner or later reach the problem of unveiling the female sex, which they find to be itself a veil - perhaps from the anxiety that its final unveiling would reveal that there is nothing to unveil. $^{9}$

Le plus important ici est la similarité entre la pudeur des réalistes et l'échange de regards qui produit l'effet de la pornographie selon Gade. Il met en avant que le rôle incitant du regard remonte au premier érotisme visuel qui parut avec la naissance de la photographie, à la même époque que l'écriture des œuvres réalistes ; une époque où la vue était la garantie première de la vérité. Dans ce contexte, on peut considérer les descriptions du corps chez Flaubert et Zola comme autant de variations sur les limites et les possibles du regard. Si les réalistes s'intéressent au corps, ce n'est pas d'abord comme motif ou comme mode d'être, mais comme endroit où l'économie de la représentation est mise en évidence. La description du corps est en même temps la représentation du but du regard désirant et la réflexion du regard contemplatif en regard du motif, et permet ainsi la mise en scène du désir lui-même. Mais le corps lui-même devient alors indescriptible, puisqu'une telle description démantèle le circuit des regards nettement équilibré et laisse le corps dénudé, sans intérêt. Le titre esthétique du réalisme est exactement l'effet de cette distanciation du motif, le résultat d'une discrétion dans la description qui est un précurseur de mise en scène efficace du désir dans la pornographie. Le réalisme, tout comme la pornographie, est soumis à un paradoxe insoluble, dans lequel le désir d'explicitation s'oppose à la nécessité esthétique et sexuelle d'une distanciation pour soutenir le désir; exactement comme Rudi en fait l'expérience dans Lanzarote.

\footnotetext{
${ }^{8}$ Émile Zola, Nana, Paris, Classiques Garnier, 1994, p. 177.

${ }^{9}$ Peter Brooks, Body Work - Objects of Desire in Modern Narrative, Cambridge, MA/London, Harvard University Press, 1993, p. 159. Je me contente ici d'étudier les aspects techniques de la description, excluant volontairement l'interprétation freudienne du fétichisme que propose Brooks, malgré les perspectives intéressantes qu'elle ouvre.
} 


\section{Vers un érotisme non visuel}

Le paradoxe de la visualité est parfaitement clair dans l'œuvre de Houellebecq, et c'est peut-être pour cela, que l'on peut y voir plusieurs tentatives de créer des descriptions du corps qui s'échappent de ce dilemme. Un bon exemple est la scène dans Les Particules élémentaires où Bruno rencontre Christiane pour la première fois dans un jacuzzi. Les premières lignes de la description, qui se poursuit sur plusieurs pages, nous suffisent :

\footnotetext{
Elle allongea les jambes dans l'eau. Bruno fit de même. Un pied se posa sur sa cuisse, frôla son sexe. Avec un léger clapotis, elle se détache du bord et vint à lui. Des nuages voilaient maintenant la lune ; la femme était à cinquante centimètres, mais il ne distinguait toujours pas ses traits. Un bras se plaça sous le haut de ses cuisses, l'autre enlaça ses épaules. Bruno se blottit contre elle, le visage à hauteur de sa poitrine ; ses seins étaient petits et fermes. ${ }^{10}$
}

Le contraste avec la scène pornographique de Lanzarote est frappant. Si l'épisode avec les deux lesbiennes était basé sur une visualité absolue, tout était visible, dans cette scène-ci Bruno ne voit rien : le plus important est immergé, il fait nuit, et au moment où Christiane se penche sur lui, un nuage couvre la lune pour absorber le dernier résidu de lumière. Ainsi leur rencontre sexuelle n'est-elle pas l'effet d'une stimulation visuelle du désir mais le produit du plaisir que procure le toucher. Cette relation se reflète dans la description de la scène. Le rythme de la narration est nettement plus lent par rapport à la description de la scène sur la plage, qui était forcée, centrée sur les échanges d'actes sexuels. Ici, la description suit patiemment les attouchements, le pied sur sa cuisse, le frôlement de son sexe, le clapotis de l'eau, le bras sur ses cuisses etc.. Le rythme de la lecture et de la description s'unissent pour donner à la scène une plénitude sensible, vivifiée par le temps que se donne le lecteur pour parcourir le texte. Houellebecq essaie de capter la matérialité temporelle du texte en laissant les attouchements et la tendresse de Christiane mener la narration. La description de la rencontre de Bruno et de Christiane se distingue ainsi de la plupart des descriptions sexuelles dans les romans de Michel Houellebecq. Le passage cité évoque une tendresse qui sera à l'origine de l'amour entre Bruno et Christiane. Mais cet état de plaisir pur ne se

\footnotetext{
${ }^{10}$ Michel Houellebecq, Les Particules élémentaires, Paris, 1998, J 'ai lu, p. 138.
} 
maintient pas. Comme il a été dit plus haut, leur liaison est marquée par la poursuite de stimulations toujours plus fortes, une poursuite qui se termine par la dépression de Christiane puis son suicide subséquent. Le plaisir pur est et reste une exception, qui avec le temps s'engouffrera dans le vortex du désir; comme dans le cas de Rudi dans Lanzarote.

De la même manière, la patiente description à laquelle s'essaie Houellebecq n'est pas une réelle alternative à la description réaliste, mais une exception provisoire. Le passage cité désavoue le regard, mais est néanmoins basé sur une structure de visualité. Bien que les deux personnages soient cachés par l'obscurité, les signes visuels constitutifs de la sexualité, seins et sexes, sont parfaitement clairs dans la description et organisent la narration, ce qui met en évidence que la rencontre de Bruno et Christiane n'est pas la redécouverte d'une sexualité pré-visuelle, mais au contraire la mise en scène de la sexualité visuelle. L'aventure dans le spa est particulièrement excitante parce qu'elle implique la conscience de la suspension de la visualité. La confirmation du désir par le regard est impossible de sorte que l'obscurité elle-même devient une confirmation du désir de la femme et de la puissance de l'homme.

Cette dynamique est ègalement évidente dans un passage du texte «Cléopâtre 2000 », qui décrit une sortie de Houellebecq et de sa femme, Marie-Pierre, dans une boîte échangiste, Cléopâtre, sur le Cap d'Agde. Le point culminant de la visite est un glory hole, un trou par lequel l'homme passe son pénis pour être satisfait anonymement, sans qu'il puisse voir la personne de l'autre côté ${ }^{11}$. Aussi bien ici, que dans le spa, la satisfaction n'est pas directement liée à la visualité, mais indirectement parce qu'on imagine ce qui pourrait être de l'autre côté. Ainsi, la structure schématise le scénario pornographique dans sa forme la plus pure. Le phallus se substitue à l'homme, le regard de la femme est intériorisé dans la conscience de l'homme, et l'attouchement du pénis confirme ce regard, comme la preuve d'un regard désirant capté par le phallus de l'autre côté du mur. Dans le jacuzzi tout comme à la Cléopâtre, l'expérience sexuelle qui serait purement tactile et sensuelle, est en réalité dépendante d'une culture visuelle, tout comme la description que fait Houellebecq de la scène. C'est justement cette cécité qui permet au narrateur de transférer ses

${ }^{11}$ Michel Houellebecq, Lanzarote et autres textes, op. cit., p. 82. 
propres conceptions de la pureté du désir à l'autre, de sorte que l'attouchement réel, la véritable rencontre entre deux personnes, s'efface. La description tactile, sensuelle, dépend des mêmes procédés que la pornographie et court le risque de représenter l'idéal d'un désir pur, plutôt que de décrire la sensualité dans son apparition. Retrouver une innocence perdue est un projet oxymorique qui s'appuiera toujours sur les prémisses de la culpabilité et de la conscience.

\section{Le mécanicité du paysage}

Si la description d'une sexualité pure n'est pas une réelle alternative, Houellebecq porte toutefois un coup bien plus radical à la visualité, sapant ses fondements, dans une série de paysages. La possibilité d'un tel sapement apparaît dans ce passage de la fin de Lanzarote, où le narrateur quitte ce qu'on pourrait appeler le véritable personnage principal du récit : l'île elle-même et son paysage volcanique millénaire :

Au moment du décollage, je jetai un dernier regard sur le paysage de volcans, d'un rouge sombre dans le jour naissant. Étaient-ils rassurants, constituaientils au contraire une menace ? Je n'aurais su le dire ; mais quoi qu'il en soit ils représentaient la possibilité d'une régénération, d'un nouveau départ. Régénération par le feu, me dis-je. ${ }^{12}$

Houellebecq propose ici une autre possibilité pour se dégager de l'histoire de la représentation; mais cette fois-ci ce n'est pas sous les douces formes de la sensualité, mais sous les contours inexorables des roches volcaniques : la régénération par le feu.

Lanzarote n'est pas la destination touristique la plus évidente. Les plages sont belles et le climat y est doux, mais la majeure partie de l'île est recouverte d'un paysage impitoyable, «d'une brutalité totale $^{13} »$, qui résiste à l'industrie du tourisme qui cherche à le domestiquer et le commercialiser. Un paysage insoumis que personne n'a façonné et qui ne laisse aucune place à la vie, mais suspend tout ordre, tout discours qui essaierait de le saisir. Cela explique sans doute pourquoi Houellebecq ne tente pas de décrire visuellement le paysage, mais focalise la narration sur les occupations absurdes des personnages et le contraste avec l'environnement dans lequel ils évoluent. C'est uniquement dans une série de photographies, prise par Houelle-

${ }_{12}^{12}$ Michel Houellebecq, Lanzarote, op. cit., pp. 74-75.

${ }^{13}$ Ibid., p. 23. 
becq, qui accompagne l'édition que nous trouvons une représentation directe du paysage volcanique. Les photos ne sont pas artistiques, mais sont composées de paysages simples, montrant les formations volcaniques qui donnent à l'île un caractère lunaire et hostile. Ce n'est pas la grandeur du paysage qui est au centre mais le rocher et sa formation géologique que marque le paysage. Les photos de Houellebecq ne représentent pas le paysage comme un ensemble intelligible mais rendent compte avec exactitude de la structure du rocher grâce à la mécanique de la caméra.

Cette approche du monde s'oppose de manière significative à différentes sortes de descriptions du corps humain mentionnées plus haut. Quoiqu'il s'agisse de photos, la représentation n'est pas de nature visuelle. Les photos ne sont pas structurées pour la vue humaine, mais sont des indices, des empreintes de la réalité présente, qui suivent leur structure. Il n'y a pas de construction de perspective cavalière, au contraire, la structure stratifiée de la roche subvertit toute tentative de vue d'ensemble. L'observateur est obligé de suivre la structure du rocher pour percevoir l'image, ce qui rend le procès de voir lui-même tactile. La vue, le regard change de caractère et n'y est pas une manifestation du désir totalisant, mais une contemplation désintéressée qu'observe l'objet avec humilité, qui fait la matière expressive elle-même : l'immobilité du rocher forgé par l'explosion volcanique rend le paysage expressif, non qu'il nous parle, mais il murmure doucement en lui-même l'histoire du temps où la lave coulait à flots.

Ces descriptions d'une nature non humaine se fondent dans la description du corps dans les utopies qui imaginent le développement de l'espèce humaine, comme celles que l'on trouve à la fin des Particules élémentaires et surtout dans La Possibilité d'une île. Dans ces deux romans, Houellebecq décrit une société du futur où les progrès de la technologie ont rendu possible la modification de l'espèce humaine. Le philosophe allemand Peter Sloterdijk a construit le concept de Homöotechnik, qui désigne les capacités de la technologie nouvelle à modifier le corps humain; les technologies nouvelles inscrivent l'information dans le corps lui-même et le rendent ainsi expressif d'une autre manière : 
Die Homöotechnik hingegen kommt, weil sie es mit real existierender Information zu tun hat, nur noch auf dem Weg der Nicht-Verwaltigung des Seienden voran; sie greift Intelligenz intelligent auf und erzeugt neue Zustände von Intelligenz; sie hat Erfolg als Nicht-Ignoranz gegen verkörperte Information. ${ }^{14}$

L'intelligence incorporée a une fonction immanente, et non pas transcendante, en la relation aux autres intelligences, ce qui règle le problème de la représentation effectivement. Puisque le corps luimême devient l'intermédiaire par clonage ou par mémorisation directe d'informations par le cerveau, la primauté de la vue est levée, en faveur d'une transmission ouverte d'informations. Ainsi, la révolution homöotechnologique représente une réelle possibilité de libération, comme le montre Les Particules élémentaires. L'espèce humaine nouvelle mène une vie heureuse dans laquelle tous les désirs sont instamment satisfaits par l'effet d'une quantité augmentée de corpuscules de Krause, ce qui rend possible : " des sensations érotiques nouvelles et presque inouïes ${ }^{15} »$. L'économie sexuelle devient inutile à cause de la modification du corps humain et du dépassement du paradoxe réaliste du désir qui est inscrite dans la chair même, dans la séquence ADN de l'homme.

Cependant, le dernier roman de Houellebecq, La Possibilité d'une île, peut être conçu comme un correctif à cette conception optimiste des possibilités de la technologie. Dans ce roman aussi, une espèce nouvelle fondée sur le clonage est créée mais cette fois pour recevoir les âmes d'individus qui existent déjà. Quand les individus atteignent un certain âge, on les transfère dans un nouveau corps déjà adulte. Un procédé qui peut être répété à l'infini rendant l'homme immortel. Ce transfert nécessite toutefois que la mémoire et les pensées du mourant puissent être communiquées au nouveau corps ; un procédé qui, dans le roman, se trouve être impossible. À titre alternatif, les nouveaux corps sont munis de rapports écrits sur la vie des générations précédentes, pour assurer une sorte de continuité au-delà de la continuité physique. Néanmoins, ce développement souligne un point très important : si le corps est modifié et devient de ce fait expressif, la relation entre corps et âme est bouleversée. Le corps n'est plus le récipient de l'âme, mais la chose même, et n'est par conséquent plus le corps dans

\footnotetext{
${ }^{14}$ Peter Sloterdijk (2001) Das Menschentreibhaus - Stichworte zur historischen und prophetischen Anthropologie, Weimar, Verlag und Datenbank für Geisteswissenschaften, p. 73.

${ }^{15}$ Michel Houellebecq, Les Particules élémentaires, op. cit., p. 312.
} 
le sens où nous l'entendons. Michel Serres a décrit comment les technologies nouvelles transforment le corps en « un virtuel incarné ${ }^{16}$ ». Le corps cesse d'être un objet pour se faire virtualité qui s'exprime, non pas en langage, mais en codes : pointures, tour de poitrine, mots de passe, mais surtout et avant tout en codes ADN. Le corps est écrit et s'écrit dans le langage simple des mathématiques, ce qui marque la transition vers une visualité fragmentée, qui néanmoins élève la vue à sa plus haute puissance. La métamorphose est manifeste dans La Possibilité d'une île, où l'espèce nouvelle a à peu près renoncé son existence physique en faveur d'une vie solitaire dans laquelle l'unique contact avec le monde extérieur s'opère par médias visuels et le désir sexuel est presque éteint. Il ne reste que des souvenirs écrits du désir, souvenirs qui en soi peuvent exciter des sentiments : «Ce qu'elles avaient par contre connu, et cela de manière singulièrement douloureuse, c'était la nostalgie du désir, l'envie de l'éprouver à nouveau, d'être irradiées comme leurs lointaines ancêtres par cette force qui paraissait si puissante ${ }^{17} »$. Le résultat n'en est pas un érotisme nonvisuel, mais au contraire une visualité sans désir, dont l'expérience se révèle insupportable. La fin du roman oppose ainsi les abstractions pures que sont les néo-humains et la corporalité pure des humains restants, les sauvages, une opposition qui culmine, quand le narrateur Daniel25 tente d'avoir des relations sexuelles avec une jeune sauvage mais renonce, repoussé par: «l'odeur pestilentielle qui émanait de son entrecuisse ${ }^{18} »$. Cette scène comprend le contraste entre sexualité prévisuelle et la visualité postsexuelle, comme Houellebecq l'a mis en scène dans La Possibilité d'une île, et indique ainsi, que ce paradoxe fondamental combattu par les réalistes du XIX ${ }^{\mathrm{e}}$ siècle est toujours intact. Daniel25 arrive à la même conclusion, quand il atterrit sur l'île de Lanzarote dans les derniers chapitres du roman. Si dans Lanzarote l'inhumanité volcanique de l'île représente pour le narrateur un impératif : renaissance ou mort; dans La Possibilité d'une île, l'île est un endroit tranquille, qui expose dans sa monotonie l'impossibilité du bonheur. Néanmoins, le narrateur trouve, dans cette absurdité absolue, une gravité dont sa non-corporalité jusqu'ici l'avait dépourvu. Seulement dans cet état, entre corps et non-corps, entre vie et non-vie, le

\footnotetext{
${ }^{16}$ Michel Serres, Hominescence, Paris, Éditions Le Pommier, Le Livre de Poche, 2001, p. 50.

${ }^{17}$ Michel Houellebecq, La Possibilité d'une île, Paris, Fayard, 2005, p. 425.

${ }^{18}$ Ibid., p. 460.
} 
narrateur peut être. La fin du roman pose peut-être ainsi une solution au paradoxe de la représentation : le maintien du paradoxe : «J'étais, je n'étais plus. La vie était réelle ${ }^{19} »$.

${ }^{19}$ Ibid., p. 485. 